\title{
XVIII.
}

Mittheilungen aus einer zwanzigjährigen Praxis in New-0rlrans. Von

M. Schuppert. M. D.

\section{A. Nervenkrankheiten.}

Zwei Fälle von rechtseitigem Gesichtsschmerz. Dolor. Fothergili.

1. Fall. Heilung durch Resection des Nervus facialis am Foramen stylomastoideum nach 6 vorausgegangenen, theilweise nur temporäre Hullfe bringenden Operationen, worunter Excision des Nerv. maxillaris super. am Foramen rotundum von der Fossa sphenomaxillaris aus, ferner partielle Resection des Nerv. maxillaris infer. nebst Unterbindung der Arteria carotis com. dextra.

A. Morris, ein Jude aus Polen, 34 Jahre alt, von kräftigem Körperbau, war im Jahre 1858 hier eingewandert; er betrieb das Geschäft eines Hausirers in Schnittwaaren und hatte sich, wie er glaubte, dureh eine Erkältung vor zwei Jahren einen rechtseitigen Gesichtsschmerz zugezogen. Die Schmerzen mussten seiner Beschreibung nach sehr heftiger Natur sein. Im Glauben, dass dieselben ihren Sitz in den oberen Backzälnnen hätten, waren dieselben einer nach dem andern geopfert worden, ohne jedoch die geringste Erleichterung zu erzielen. Patient war bereits in den Händen der verschiedensten Heilkünstler gewesen und ich hatte demnach Ursache zu vermuthen, dass der Arzneischatz so ziemlich erschöpft sei. Mit seiner Einwilligung unternahm ich daher eine auf die Dauer zwar nicht viel versprechende, aber den Umständen angemessene und für den Anfang nicht allzuabschreckende Operation. Ich machte 
eine Incision oberbali, des Areolarprocesses, wo die zwei letuten Mahlzähne gestanden, legte den Oberkiefer daselbst bloss und scarificirte denselber, machte darauf mit Huilfe einer Trephine eine Oeffnung durch dic Höhle des zweiten Mahlzahnes, gross genug, um auch die Innenfäche des Oberkiefers serarificiren zu können. Nach vollendeter Operation verschwanden die Schmerzen mehrere Monate hindurch, um danu in grösserer Heftigkeit wieder zu kebren. Diesmal verlegte Patient den Ausgangsort der Schmerzen hinter den rechten oberen Augenzahn ,und von da nach dem Auge sich erstreckend. Dieses Leiden quälte den Kranken bei Tag und Nacht; Schlucken, Bewegung der Lippen, ja ein Luftzug waren hinreichend die lieftigsten Paroxysinen beryorumufen. In nassem Wetter war sein Leiden heftiger als in trockenem. Unbelehrt durch fruhere Versuche hatte er sich abermals ein paar Zähne ausziehen lassen, natiirlich obne dadurch eine Linderung zu erzielen. Neun Monate hindurch hatte der Kranke diese Qualen ertragen, als er im Anfang September 1865 sich wieder bei mir eiustellte. Ich machte ihn damit bekannt, dass ein grösserer Nerv herausgeschnitten werden müsste, wozu er sich diesmal bereitwillig verstand. Das Antlitz des Kranken war uuunterbrochen ron Muskelzuckungen bewegt, ja im Laufe der Zeit hatte sich durch die andauernden Contractionen eine Vertiefung zwischen Levator anguli oris und Zygomaticus ausgebildet. Das rechte Auge thränte profus. Ich hoffte durch eine Resection des Infraorbitalnerven mit Einschluss der vorderen Zahnnerven die Schmexzen entfemen zu können. Am 13. September 1865 wurde diese Operation an dem mit Chloroform Narkotisirten ausgefuhht. Mit 3 Fingern der linken Hand das untere Augenlid fixirend und unterhalb desselben die Weichtheile geoen den Orbitalrand andrückend, machte jch entlang desselben einen Einscbnitt. Von der Mitte dieses Einselnittes aus erstreckte sich ein zweiter perpendiculärer Schnitt nach unten uiber das Foramen infraorbitale hinaus, $15 \mathrm{Mm}$. in Länge. Nach Ablösung der Integumente des Bodens der Augenhölule mit Einschluss des geschonten Periosts, wurden dieselbea in einem stumpfen Haken aufgenommen und mitsammt Augenlid und Augapfel emporgehoben. Die untere dünne knöcherne Lamelle der Augenhöhle wúrde mit einem Meissel bis zum Freiliegen des Infraorbitalnerven entfernt. Derselbe wurde mit einer Pincette aufgehoben, wobei die Abgänge dex vorderen Zahnnerven deutlich in Sicht kamen, hinter welchen der Nerv mit der Scheere durchschnitten wurde. Hierauf wurde das Messer in den perpendieulären Sehnitt eingesetat, der Nerr aufgesucht, freigelegt, nahe 
der Infraorbitaloffnung durchschnitten ,und aus dem Kanale herausgezogen. Die Länge resselben betrug $13 \mathrm{Mm}$. Die Blutung wal gering, kein Gefäss wurde unterbunden. Die obere Wunde wurde nit Knopinäthen, die untere mit Karisbader Naleh geschlossen. Nach dem Erwachen klagte I'atient aber "schiessende Schmerzen" in der Wange. Es wurden Eisumschläge verordnet und Abends ein Opiat verabreicht. Die Nacht verbrachte er schlaflos, durch 1/4-stindliche wiederkehrende Paroxysmen gemartert. Am nächsten-Morgen war sein Gesicht beträchtlich angeschwollen, die Augenlider geschlossen. Es wurde cin Purgans von schwefels. Magnesia gegeben. Am 15. hatten sich die Schmerzen vermehrt, die Anschwellung hatte abgenommen. 16. keine Veränderung, die Suturen wurden entfernt, die Wunden waren per primam intent. vereinigt. 17. Schnerzen wathrend der verflossenen Nacht heftig, die Conjunctiva des Auges und unteren Augenlides blutig infiltrirt. Patient hatte ausser einer Tasse Thee kein Nahrungsmittel während dieser Tage zu sich genommen. 18. Schmerzen in halbstiindlichen Intervallen sich wiederholend. Die rechtseitige Oberlippenhälfte, der rechte Nasenflügel, das untere Augenlid, und der grössere Theil der Wangengegend waren vollkommen anästhetisch, wie auch die denselben zugehörenden Schleimhäute. Eine gleichzeitige Einbusse der Motilität war augenscheinlich. Am Abend erhielt Patient cine Dosis Chinin (gr. $\mathrm{x}$ ) mit Opiun (gr. 11). El hatte nur 3 Anfälle während der Nacht und schlief zum ersten Mal seit der Operation mehrere Stunden hindurch. Am 20. wurde die Arznei wiederholt. Die Nacht verlief besser als die vorhergehende, auch traten während des 'Tages nur wenige Paroxysmen auf. Diese Besserung war zweifellos dem Opium znzuschreiben, da mit dem Wegfall desselben die Schmerzen sich vermehrten. Ich war von der Nothwendigkeit einer ausgedehnteren Operation iiberzeugt und bescbloss die Resection des obcren Maxillarnerven am Foramen rotundum selbst mit Einsebluss des Meckel'schen Ganglions vorzunehmen. So weit meine Erfalurung reichte, waren zur Zeit uur 3 Fälle von totaler Resection des zweiten Astes des Quintus bekannt und sämmtliche Fälle waren von Dr. Carnochan in New-York operirt worden. Ehe ich mich entschloss, die Operation auszufuhren, schrieb ich an Carnochan und bat um Auskunft uber die Endresultate seiner Operationen und erhielt eine sehr aufmunternde Antwort. Die Methode Carnochan's bestand darin, dass er die Oberkieferhöhle unterhalb des Augenhöhlenrandes offnete und den Infraorbitalnerven zum Leiter nehmend die hintere Wand der Highmorshöhle perforirte, dann den Nerven an Foramen 
rotundum absehnitt und denselben herauszog. Da ich den Infaorbitalnerven bereits grisstentheils entfernt hatte, musste ich desselben als Leiter cntbehren. Dem Kranken die Opcration an cinem Schidld henonstritend, sal ich beim Durchfuthen der Sonde dureh den Infranrbitalkanal, dass dieselbe in der Sphenomaxillargrube 3-4 Mn. frei lag und daran reihte sich in natiirlicher Gedankenfolge die Frage ïber die Möglichkeit, die Operation an dieser Stelle ausfiuhren zu können. Es cntstand die Frage, wie Raum gewinnen für cine so delicate Operation, wie die Resection eines Nerven, eingebettet in solcher Tiefe und in so enger Grube, dass nur einige Millimeter des Nerven zum Vorschein kamen; wo iberdies das Wangenbein mit dem Fortsatz des Schläfenbeins das Ope-rationsfeld ubcrbrickten, wo der Processus coronoideus des Unterkiefer's mit seinem mächtigen Temporalmuskel sich iiber den Nerven hinlegten und wozu sich noch das ïberaus fleischige Gesicht des Kranken gesellte, um die Tiefe, in welcher der Nerv aufzusuchen, zu vermehren. Allein gerade diese Hindornisse, verbunden mit der Neuheit des Gedankens, waren anspornend genug, die Idee zur Ausführung zu bringen. Die knöcherne Brücke kam ja wenig in Betracht, sie liess sich lejeht entfernen, und das Hinderniss, welches in dem Processus coronoideus des Unterkiefers lag, konnte durch Oeffnen des Mundes beseitigt werden; so blieb denn nur als Hinderniss die Enge der Kieferkeilbeingrube, und auch diese konnte crweitert werden durch ein Wegmeisseln der hinteren dïnnen Wand des Oberkiefers. Was endlich die möglicherweise eintretende Entstellung des Gesichts betraf, so kam dieselbe bei der Heftigkeit der Schmerzen kaum in Betracht, wurde auch durch die Einwilligung des Kranken zu der vorzunehmenden Operation vollkommen beseitigt. Am 25. September $12 \mathrm{Uhr}$ Mittags in Gegenwart der Doctoren Barnes, Eylandt, Goldmann, Hassenburg, Loeber, Sehmittle u. A. wude Patient chloroformint. Derselbe lag in einem Sessel mit beweglicher Rückcnlehne, in einer zweckmässigen Lage, das Operationsfeld von der Sonne beschienen. Nahezu 3 Unzen Chloroform waren verbraucht, ehe die nöthige Narcotisation erreicht war. Ein Einschuitt durch die Haut, in zwei Centimeter Entfernung von dem äusseren Winkel des rechten Auges und in der Höhe der Augenbraue beginnend, wurde in einer nach aussen convexen Linie nach unten gefibrt, so dass er gegeniber dem unteren Rande des Nasenflügels in 4 Centimeter Entfernung von dem Mundwinkel endigte. Die Länge des Sclnittes betrug 8 Centimenter, Blutung war geriug, kein Gefäss wurde unterbunden. Die Weichtheile uiber dem 
Wangenbein und dem Jochfortsatz des Schlätenbeins wurden abgelöst bis zu den Stellen, woselbst die Knochen mittelst der Liston'schen Zange durchschnitten werden sollten. Ein Zangenschnitt befand sich in der Nähe der Vereinigung des Wangenbeins mit dem Jochfortsatz des Schläfenbeins, wodurch ein Drittheil des Wangenbeins getrennt wurde, der andere entfernte den grössten Theil des Jochfortsatzes; der an dem resecirten Knochensticke befestigte Masseter wurde abgelöst. Es war hiermit ein Raum gewonnen, um zwei Finger einftihren zu können. Hierauf wurde das Messer bis auf den Knochen des Oberkiefers eingestossen und entlang der Oberkieferkeilbeingrube geführt, um die sehnigen Fasern des äusseren Flügelbeinmuskels zu durchschneiden. Das Messer wurde darauf umgekehrt und mit dem spadelförmigen hölzernen Ende die Entblössung des Knochens vollendet. Die losgelösten Muskelfasern wurden mit einem stumpfen Haken nach aussen gehalten. Der Processus coronoideus des Unterkiefers war der Ausfihrung der Operation nicht im Wege, allein eine Portion des an ihn befestigten Temporalmuskels musste getrennt werden. Hierauf wurde das Messer gegen zwei Pineetten vertauscht; bei der Herausnahme einer Portion Fett wurden einige Venen des Plexus pterygoideus zerrissen, eine starke Blutung veranlassend, welche dureh Druck mit Schwämmchen und Eis gestillt wurde. Dieser Zwischenfall verzögerte die Operation beträchtlich, da es unmöglich war, den Nervon aufzufinden, so lange die Blutung anhielt. Die Wunde hatte hier eine Tiefe von 6 Centimetern erreicht. Die Art. maxillaris inter. konnte deutlich im unteren Ende der Wunde gefiihlt werden, war jedoch gänzlich ausserhalb des Bereiches einer Verletzung. Nach Stillung der Blutung wurde das Aufsuchen des Nerven wieder vorgenommen. Ich glaubte zu verschiedenen Malen denselben gefasst zu haben, aber es waren weisse sehnige Fasern des Flïgelmuskels. Endlich gelang: es des Nerven habhaft zu werden, und wurde derselbe von allen Anwesenden als der wahre Jacob erkannt. Derselbe war indess unbeweglich. Ich beschloss deshalb, was ich schon längst hätte thun sollen, einen Theil der hinteren ()berkieferwand wegrumeisseln, wodurch der Nerv freigelegt und beweglich wurde. Ich hatte ungefähr 1 Centimeter von dem Knochen weggenommen, mit der Vorsicht jedoch, die innere Schleimhaut der Oberkieferhöhle nicht zu verletzen, als die hinteren Zahnnerven zu Gesicht kamen. Dieselben wurden getrennt und hinter ihnen der Hauptnerv durchschnitten; hierauf nach dem Foramen rotundum mich wendend, wurde der Oberkiefernerv auch hier mit sammt seiner Nachbarschaft, dem 
Meckel'sehen Ganglion entfernt. Da keine weitere Blutung eintrat, wurde die Wunde mit $>$ Karlsbader Nadeln geschlossen. Am unteren Ende der Wunde wurde eine Oeffnung für die Drainage gelassen. Die Operation dauerte nahezu $11_{2}$ Stunden und es waren 3,4 Pfund Chloroform verbraucht worden (ein grosser Theil desselben war verschwendet worden, da ich stets die Vorsicht anwende, das Chloroform mit einer gehörigen Quantität atmosphärischen Luft gemischt zu verabreichen). Im vollen Vertrauen, dass der Kranke durch diese Operation von seinen Schmerzen fur immer befreit sein wiirde, wartete ich dennoch mit einem peinlichen Gefühl auf sein Erwachen, um aus seinem Munde die frohe Kunde zu vernehmen, dass sein alter Peiniger verduftet sei. Es vergingen zwei unendlich lange Stunden, ehe der Operirte sich so weit erholt hatte, um den noch Gegenwärtigen mittheilen zu können, dass seine alten Schmerzen ihn verlassen hätten. Mehrere Tage hindurch fühlte sich Pat. sehr unwohl, er brach häufig und konnte, wie er sagte, den Geruch des Chloroforms nicht los werden. Nun er hatte freilich eine gute Dosis zu sich genommen. Fünf Tage hindurch wurden Eisumschläge angewandt, als sich eine Gesichtsrose einstellte, wo alsdann die kalten Umschläge gegen warme vertauscht wurden. Die Nadeln waren schon am 3. Tage herausgenommen, die Wunde war per primam intentiom geheilt. Am unteren Mundwinkel musste dieselbe wieder eröffnet werden, um ein Drainagerohr einzulegen, da sich eine ziemliche Quantität Eiter angesammelt hatte. Die Operation hatte auf das arterielle System keinen Einfluss ausgeübt. Der Puls zeigte 80 Schläge in der Minute vor wie nach der Operation und veränderte sich auch nicht während der eintretenden starken Eiterung. Patient war sehr enthaltsam, während der ersten 8 Tage nacb der Operation batte er mit Ausnahme einer Tasse Kaffee mit Milch nichts genossen. 14 Tage später war das Gesicht wohl noch etwas geschwollen, die Wunde eiterte gering, aber die Hauptsache, seine alten Schmerzen, waren nicht wiedergekehrt. Am 29. October war das untere Aug'enlid noch ein wenig ödematös, das Kauen minder beschwerlich, er konnte den Mund zur Hälfte seiner Ausdehnungsfähigkeit öffnen, die Wunde war geschlossen, die Eiterung hatte fast gänzlich aufgehört. Die Anästhesie, welche nach der vorigen Operation eingetreten war, hatte durch diesen operativen Eingriff keine Gebietserweiterung erhalten. Zwei Monate nach der Operation ging I. seinen Geschäften wieder nach und vor seiner Abreise nach Texas erklärte er ein anderer Mensch zu sein. Das Masticationsvermög'en war vollständig hergestellt, eine kleine Fistel 
in unteren Wundwinkel sonderte noch einige Tropfen einer serïsen Flïssigkeit ab, das Gesicht zeigte eine geringe Difformität. Die rechte Gesichtshälfte war vollständig anästhetisch, wie anch eine partielle Lähmung derselben noch vorhanden war. Leider sollten meine sanguinischen Hoffnumgen, den Feind fiur immer schadlos gemacht zu haben, nicht in Erfiillung gehen.*)

Von Texas zuriickgekehrt crschien Morris in meiner Office im Mai 1867, mir mittheilend, dass er während der zwei letzten Monate wiederum heftige Schmerzen ausgestanden, ja dass er während dieser Zeit keine Mahlzeit zu sich genommen, ohne an sein altes Leiden erinnert worden zu sein. Der Sitz der Schmerzen war der alte, im Oberkiefer, die Paroxysmen gingen von der rechten harten Gaumenwand aus nach der Schläfe und lem Ange sich lin erstreckend. Die Sensibilität sïmmtlicher Gesichtstheile, welche durch eine vorausgegangene 0 peration zerstört worden, war voll kom m e n wieder hergestellt. Patient fühlte eine Nadelspitze gegen das untere Augenlid, den Nasenflugel oder die Oberlippe gehalten, wie auf der gesunden Seite. Mit dem Verschwinden der Anästhesie waren nach Aussage des Kranken die Schmerzen zurückgekehrt. Ich war mir bewusst, den mittleren Theil des Oberkiefernerven bei der letzten Operation nicht entfernt zu haben, da ich den Nerven hinter dem Abgang des hinteren Zahnnerven abgeschnitten hatte. Ueber den Grund dieses Versehens konnte ich mir keine Rechenschaft geben. Ich bereute dies bitter, da ja möglicherweise mit diesem zuriickgebliebenen Nervenrest der Schmerz in Verbindung stehen konnte. Es sehwebte mir bei dieser Annahme das Gespenst der Nervenregeneration vor Angen, von welchem ich freilich in meiner Praxis nie etwas gesehen, desto mehr aber dariiber gelesen hatte, und zwar von glaubwiurdigen Autoren, welche eine Wiederkehr der Schmerzen nach Nervenresectionen aus einer Regeneration derselben erklärten. Ich war genöthigt, dem Patienten meinen Fehler einzugestehen und eine Wegnahme des zuräckgelassenen Nervenrestes vorzuschlagen, indem ihm dadurch vielleicht Hülfe geleistet werden könne. Morris war dazu bereit und so wurde derselbe am 2. Juni wiederholt chloroformirt und die 4.Operation unternommen. Ich hatte in dem vorliegenden Falle keine andere Wahl, als die Car-

*) Einige Jahre nach stattgefundener Resection des Oberkiefernerven in der Oberkieferkeilbeingrube, erfuhr ich, jass Prof. Bruns in Tibingen diese Methode bereits am Cadaver demonstrirt habe. In der Wahl zwischen dieser und der Methode des New.Yorker Chirargen Carnoehan muss ieh indess letzterer den Vorzug geben. 
nochan'sche Methode zu adoptiren. Die Narbe am unteren Orbitalrancle, wolche ron der zweiten operation berstammte, wurde durehschnitten, der frei geleyte Orlitalrand wit der Stichsäge an zwei Punkten greich weit ron der litte entfernt durehsehnitten und das hierdurch gebildete knochenstick mit einer Knochenzange entfernt. Der Buden der Augenhöhle, dessen diinne Knochenlamellen in der frïheren Operation entfernt worden waren, hatte sich durch eine neue dicke Knochennasse ersetzt und musste mit dem Meissel hinweggenommen werden. Hatte hier durch das erhaltene Periost eine Knochenneubildung stattgefunden, so war dagegen von einer Regeneration von Nervenfasern nicht die Spur wahrzunehmen, ebensoweng hier wie in der unteren porpendicularen Narbe, welche ich zu dem Zwecke durchselnitt, un mich hier gleichfalls von der Anoder Abwesenheit eines regenerirten Nerven zu iaberzeugen. Was bei dem Erüfnen der Narben auffiel, war die durch eine starke Blutung sich manifestirende Vascularität der Gebilde, wclehe zuror wenig oder kaum geblutet hatten. In der Mitte der Orbita fand ich den gesuchten Nervenstumpf mit Nervenfasern als Anhängsel, welche den mittleren Kahnnerven angehören mussten. Der Nervenstumpf wurde lcicht herausgezogen, seine İiinge betrug $10 \mathrm{Mm}$. Derselbe zeigte an keinem seiner Enden die geringste Anlange einer Nervennoubildung. Un rollkommen sicher zu gehen, dass ich in dem Infraorbitalkanal keine Nervenfasem zurickgoelassen, führte ich (höchst überfluissiger Weise) das Gluihcisen ein, welches ich bis zur hinteren Wand des Oberkiefers wirken liess. Die Wunden wurden durch Nïbe geschlossen. Nach vollendeter Operation gerieth der Oberkörper des Patienten mit Einschluss der Extremitäten in eine zitternde Bewegung, welche eine volle stunde hindurch anhielt. Mit dem Aufhören derselben beim Erwachen des Kranken klagte derselbe über einen fromden, jedoch sehr heftigen Schmerz in seinem Kopte (sjeherlich durch das Gliiheisen hervorgebracht), welcher beinahe 4 Stunden lang anhielt. Am uächsten Morgen war das Gesicht angeschwollen und er klagte wiederum ïber seinen alten Schmerz in larten Gammen. Diese Operation war also gänzlich nutz- und effectlos gewesen, weder fand eine Abnahme der Schmerzen statt, noch wurde dalureh die Sensibilität der betreffenden Gesichtspartien ver:indert. Am 19. Juni, also 17 Tag'e nach der Operation, hatten die Schmerzen cine so unerträgliche Höhe erreicht, lass der Gemarterte dringend um eine neue Operation nachsuchte. Die Schmerzregion hatte an Ausdehnung zugenommen. Der 3. Ast des Quintus, der Inframaxillaris, war in Mitleidensebaft gezogen und 
zwar so stark, dass selbst die alten Schmerzen in den Hintergrund traten. Die geringste Beriihrung der Unterlippe, Druck gegen das Kinn riefen Schmerzparoxysmen hervor. Ich beschloss demnach einen Theil dieses Nerven zu reseciren. Patient wurde mit Chloroform narkotisirt. Es wurde ein Einschnitt gemacht, unterhalb des Ohrläppchens beginnend und am Rande des Unterkiefers in 7 Centimeter Länge sich hinziehend. Die Muskelansätze wurden ron dem Knochen abgelöst. Darauf wurden zwei Einschnitte mit der Stichsäge gemacht, welche den Knochen zu zwei Drittel trennten und von welchen der eine dem Winkel des Unterkiefers entsprach, der andere unterhalb des 2. Mahlzahnes sich befand. Das zwischen beiden Einschnitten liegende Knochenstiick wurde mittels Hammer und Meissel entfernt. Arterie und Nerv wurden durchschnitten und von letzterem 2 Centimeter resecirt. Die Arterie wurde unterbunden. Nach Stillung der Blutung wurde die Wunde mit Näthen geschlossen, mit Offenlassen des tiefsten Theiles für den Abfluss des Eiters. Patient erwachte aus seiner Narkose unter intensiven Schmerzen und Convulsionen, welche den ganzen Körper erschütterten. Dieser Zustand hielt einige Stunden hindurch an. Die Schmerzen wurden dann geringer und traten in längeren Intervallen auf. Abends wurde eine Dosis Chinin mit Opium gereicht. Patient schlief indess nur kurze Zeit und litt heftige Schmerzen den grössten Theil der Nacht hindurch. Die Unterlippe konnte berührt werden ohne die fruheren Paroxysmen hervorzubringen. Die Schmerzen schwanden nach und nach in dieser Gegend, dagegen erwachten dieselben in der Buccalregion und dem Oberkiefer wieder lebhafter. Unter dem täglichen Fortgebrauch des Chinins mit Opium wurde das Leiden indess erträglich und Morris verliess nach einem Monat unter grosser Besserung die Stadt.

Während der folgenden zwei Jahre sah ich ihn nur einmal, und er theilte mir mit, dass er ziemlich frei von Schmerzen geblieben sei, ohne dieselben jedoch ganz verloren zu haben. Diese Galgenfrist nahm im September $1868 \mathrm{ihr}$ Ende. Er stellte sich wieder mit seinem alten Peiniger bei mir ein, die Schmerzen waren heftiger wie je zuvor. Die Neuralgie im Gebiete des Unterkiefernerven war zwar verschwunden, dagegen dieselbe im harten Gaumen und dem bekannten Gebiete um so tobender Mit dem Oberkiefernerven, darüber konnte kein Zweifel obwalten, hatten wir abgeschlossen. Was nun thun? Guter Rath war hier theuer. Zwei Operationen standen noch in Aussicht, welche Besserung oder gar Heilung bringen konnten. Die Unterbindung der Carotis, welche einer unserer grossen 
Chirurgen als das Non plus ultra in der Behandlung dieser Neuralgie betrachtet, oder endlich, horribile dictu! die Resection des Noli me tangere so vieler Chirurgen, des Facialnerven. Die Empfehlung, welche die lnterbindung der Carotis begleitete, verleitete mich trotz der Zweifel, welche ich in ihre Wirksankeit hatte und welche sich auf ein gesundes Raisonnement stützten, dieselbe zu wählen. Patient war Alles zufrieden, auch wenn, wie er sich ausdrückte, ich ihm den Kopf abschneiden wollte, nur dass er von seinem Leiden befreit wiirde.

Ám 22. September, in Gegenwart der Doctoren Barnes, Geutebruck und Schmittle wurde die Unterbindung der Arter. carotis communis dextra ausgeführt. Ich glaube die Technik der Operation hier iibergehen zu können. Dieselbe nahm kaum 5 Minuten in Anspruch. Der Erfolg der Operation war, wie ich vorausgesehen, ein negativer. Dieselbe hatte auf das heftige Leiden der Neuralgie nicht den mindesten Einfluss. Morris verliess meine Office einige Stunden nach unternommener Operation und ging nach seiner 1/2 Meile entfernten Wohnung. Sieben Tage lang beobachtete ich denselben, auf einen Nachlass der Schmerzen hoffend, doch vergebens. Der Mann drängte und bat mich ihm Linderung zu verschaffen. Das Chinin mit Opium versagte diesmal jeden Dienst, und so entschloss ich mich denn, den letzten Trumpf auszuspielen und die Resection des Facialnerven vorzunehmen.

Am Morgen des 29. September wurde Patient in meiner Office chloroformirt. Die bei der letzten Operation genannten Aerzte waren abermals zugegen. Dr. Schmittle administrirte das Chloroform, allein schon nach 5 Minuten zeigten sich so alarmirende Symptome, dass die Narcotisation unterbrochen werden musste. Die Extremitäten der linken Seite des Kranken geriethen in convulsive Zuckungen, und diese klonischen Krämpfe waren so intensiv, dass Patient von mehreren der Anwesenden im Stuhle festgehalten werden musste. Nach Verlauf einer Viertelstunde, nachdem die Krämpfe aufgehört, wurde das Chloroform vorsichtig weiter gegeben, bis vollständige Narkosis eingetreten war. Ich machte darauf eine Incision, beginnend am unteren Theile des Processus mastoideus und führte dieselbe abwärts in der Richtung des Winkels des Unterkiefers Der Einschnitt war 5 Centimeter lang. Der breite Halsmuskel wurde durchschnitten, der untere Theil der Ohrspeicheldruse gelöst und mittelst eines stumpfen breiten Hakens zur Seite gehalten Mit Hiulfe des Zeigefingers und zweier Pincetten gewann ich hinreichende Tiefe, da ich aber den Raum dennoch zu eng fand, um 
den Nerven am Foramen auffinden zu können, so machte ich einen zweiten Sehnitt unterhalb des Ohrläppehens beginnend und $\mathfrak{u} b e r$ die Articnlation des Unterkiefers linaus gehend. Dieser Schnitt ging ebenfalls durch einen Theil der Ohrspeicheldrüse. Die Wunde war nun binreichend geräumig, um zu dem Nerven zu gelangen. Ich fühlte in der Tiefe des Schnittes eine schwach pulsirende Arterie, die Carotis externa, ich legte zwei Ligaturen um das Gefäss und durchschnitt dasselbe zwischen beiden. Es kam eine geringe Menge Blut aus dem unteren Ende, dagegen mehr aus dem oberen, ohne jedoch den arteriellen Stoss anzunehmen. Beide Enden wurden durch die Ligaturen geschlossen. Ich ging nun zwischen den Processus mastoideus und den Proc. stylomastoideus, den letzteren sehr deutlich fühlend. Indem ich ibu von seinen Muskelanbängen zu befreien suchte, entsand eine heftige Blutung, welche die Operation nicht allein verzögerte, sondern auch die Schuld trug, dass der Nerv nicht frei gelegt werden konnte, sollte dies nicht auf Kosten der Zeit geschehen. Um die Operation zu beschleunigen, griff ich daher zu einer über der Fläche gebogenen Scheere und schnitt die Umgebung des Griffelfortsatzes vollständig aus. Um indess sicher zu gehen, dass der Nerv der Scheere nicht entgangen, suchte ich denselben in dem Querschnitte von neuem auf. Der Pes anserinus des Nerven wurde hier leicht gefunden und ein Theil desselben resecirt. Es ereignete sich hierbei, dass die Temporalarterie durchschnitten wurde, wobei dieselbe Erscheinung wie bei der Carotis interna auftrat, eine Erscheinung, welche aus der Unterbindung der Carotis communis und der Communication mit der Arterie der andern Seite im Gehirn hinreichend erklärt wird. Die Operation dauerte dureh die öfteren Unterbrechungen ungewöhnlich lange. Beinalue $1 / 2$ Pfund Chloroform war verbraucht worden. Patient wurde in meiner Office auf eine Matratze gelegt, wo ich denselben während der Nacht uberwachte. Beim Erwachen konnte Patient kaum einige verständliche Worte hervorbringen. Wasser konnte er nicht schlucken. Neben einer Suppression des Harns und häufigem Eibrechen befand sich das Antlitz des Kranken in steten Zuekungen. Erst am folgenden Tage kehrte sein Bewusstsein völlig zurïck. Die Sprache stellte sich wieder ein, wenn auch noch schwerfällig, Schlucken gelang unter grosser Anstrengung, die Zuckungen hatten ganz aufgehört. Die Extremitäten waren kalt und es hatte sich eine vollkommene Hemiplegie nebst Anästhesie der linken Seite eingestellt. Der Puls war schwach, 64 Schläge, Schmerzen ausser solchen, welche durch die Verwundung gesetzt waren, nicht vorhanden. Während der fol- 
genden Nacht hub sich der luls, die Extremitäten wurden warm und die Urinsccretion hatte sich gleichfalls wieder eingestellt. Patient wurde an :R. Tage in scine Wohnung gebracht. Er erholte sich aufialend schnell nit Ausnahne der Heminlegte. An $\boldsymbol{i}$. October lösteu sich die rerschiedenen Ligaturen mit Einschluss der an der Carotis communis angelegten; Patient füllte zum ersten Male die Spitze einer Nadel an der unteren Extremitait. Er klagte iber ein zichendes Gefiuhl in seinem linkel Sehenkel, Bewegung konnte er dagegen mit keiner Extremität ausfithren. Die Muskeln der Extremitäten reagirten auf Application des Faradischen Stromes. October 10. Die Sensibilität etwas vermehrt, dabei Sehmerzen im linken Schenkel, Patient schläft gut und isst mit vermehrtem Appetit. October 20. Der Faradisehe Strom wurde täglich applieirt, unter dessen Einwirkung die untere Extremitiit sich auffallend schnell erholte, während derselbe auf den Arm keinen anderen sichtbaren Einfluss austibte, als eine grössere Beweglichkeit der Finger zu bewerkstelligen. December 15. Patient war im Stande mit Hïlfe eines Stockes zu gehen, den Arm in einer Schlinge tragend. Die Veränderung, welche seine Physiognomie erlitten, war eber eine giinstige zu nennen, verglichen mit dem Ausdruck, welcher nach Resection des Infraorbitalnerven stattgefunden hatte. Ende December verliess Morris New-Orleans um nach Texas uber zu siedelu. Sein alter Feind war endlich von ihm gewichen. Es sind seitdem mehr als 5 Jahre verstrichen, ohne dass auch nur eine Spur des alten Leidens aufufinden gewesen. Die untere Extrenität ist vollständig hergestellt, während die obere ihn noch zuweilen an ausgestandene Schmerzen erinnert.

2. Fall. Resection des rechten Oberkiefernerven nach Cal nochan's Methode, bei einem rechtseitigen Gesichtsschmerz. Heilung.

J. Kaufmann, lsraelit, 50 Jahre alt, "aus Frankreich eingewañdert, lebte seit 19 Jahren in dieser Stadt. Er betrieb das Geschäft eines Hausirers in Schnittwaaren, wie sein Glaubens- und Leidensgenosse Morris. Während der letzten 4 Jahre klagte K. iuber neuralgische Schmerzen in seiner rechten Gesichtshälfte, welche indess seit den letzten Jahren in soleh einem Maasse sich vermehrt hatten, dass er sein Geschäft aufgegeben und mit einer zahlreichen Fanilie, von dem bekannten Wohlthätigkeitssinn seiner hiesigen 
Glaubensgenossen unterstiitzt, ein erbärmliches Dasein fristete. K. beschrieb seinen Schmerz in der Nähe des rechten Mundwinkels beginnend und von da über die Gesichtshälfte sich ausbreitend, selbst die Region des Supraorbitalnerven iberziehend und an der Schläfe endigend. Ein Beriihren der Schläfe, des Augenlides oder Mundwinkels rief die heftigsten Paroxysmen hervor. Er brachte den Beginn seiner Krankheit in Verbindung mit einer Zahngeschwulst am oberen rechten Alveolarprocess. Nach Verschwinden derselben sollte der Schmerz sich eingestellt haben. Aehnlich allen solchen Fällen hatte er den Sitz der Schmerzen in die Zähne verlegt und dieselben einem nach dem anderen ausziehen lassen, bis er von seinem Irrthum, leider zu spät, überzengt wurde. Seit einigen Monaten war der Schmerz auch nach der linken Gesichtshälfte hin reflectirt, indess nur oberflächlich die Theile berïhrend und nicht wie auf der rechten Seite bis zum Knochen dringend. Essen wie Trinken, jede Bewegung der Lippen, ein Luftzug, veranlassten die Paroxysmen. Nach dem vergeblichen Gebrauche der verschiedensten Medicamente hatte er von dem Geschicke seines Glaubensgenossen Morris Kunde erhalten, und so kam er zu mir um Hülfe nachsuchend.

Am 19. Mai 1866 im Beisein der Doctoren B. Dowler, S. Choppin, Loeber und Schmittle wurde K. mit Chloroform anästhesirt und die Resection des Oberkiefernerven nach einer etwas modificirten Methode Carnochan's ausgeftihrt. Da dieselbe bereits in dem vorigen Falle beschrieben worden, so glaube ich hier einer näheren Beschreibung überhoben zu sein. Bei dem Vorgehen mit dem scharfen Meissel auf dem Grunde der Augenhöhle, um den Nerven aus seiner knöchernen Umgebung zu befreien, wurde durch Verletzen der Infraorbitalarterie eine beträchtliche Blutung veranlasst, welche indess bald mit Eis und Comprimiren mit Schwämmchen gestillt wurde. Nach Durchbrechung der hinteren Wand der Oberkieferhöhle mit dem Meissel, den Infraorbitalverven als Leiter mit der linken Hand in einer Pincette haltend, wurde so weit vorgegangen, bis das Instrument wieder auf Knochen stiess. Dies war die Schädelbasis und hier wurde der Nerv mit einem langen*geknöpften Bistouri abgeschnitten. Eine hierbei auftretende Blutung wurde mit dem Glüheisen gestillt. Ich verband mit dem Gebrauche des Glüheisens den Zweck, das Meckel'sche Ganglion zu vernichten, falls dasselbe nicht schon zerstört sein sollte. Der ausgeschnittene Nerv hatte eine Länge von 5 Centimeter im unausgedehnten $\mathrm{Zu}$ stande. Nach Verlauf einer halben Stunde, nachdem alle Blutung 
aufgehört hatte, wurde die Wunde geschlnssen, der untere Theil der Wunde communicirte mit der Mundböhle, durch welche die Eiterung ihren Ausgang fand. Beim Erwachen aus dem Narcotismus fiel Patient in wilde Delirien, so dass 3 Männer ihn halten mussten. Dieser Zustand währte ungefähr 3 Stunden. Nachdem der Kranke vollkommen zu sich gekommen, klagte er über furchtbare Schmerzen in seinem Kopfe, heftiger als er je ausgestanden. Ich war überzeugt, dass dieselben durch das Glüheisen hervorgebracht worden waren und wirde letuteres in vorkommendem Falle nie wieder so nahe dem Gehirn anwenden. Es wurden Eisumschläge verordnet und ein Opiat verabreicht. K. brachte die Nacht unter Schmerzen $z u$, obgleich letztere an Heftigkeit nachgelassen hatten; dieselben hielten mehrere Tage lang an. Das neuralgische Leiden dagegen war mit dem früheren nicht mehr zu vergleichen. Die Schmerzen machten sich in ihrer alten Bahn noch fühlbar, allein sie waren viel milder und iiberschritten nicht die Zeitdauer einiger Secunden. Die Näthe wurden am dritten Tage entfernt, die Wunden waren per primam intent. vereinigt. Die Anschwellung des Gesichts, welche sich nach der Operation eingestellt, verschwand, die Oeffinung der Wunde in der Nundböhle secernirte nur wenig und hörte in der 6 . Woche auf abzusondern. Am 1. Juli war nur eine schmale Narbe sichtbar, keine auffallende Entstellung des Antlitzes wahrnehmbar. Die Oberlippe hing ein wenig tiefer herab als auf der andern Seite. Verlust der Sensibilität erstreckte sich auf alle vom Infraorbitalis versorgten Theile des Gesichts und letztere kehrte erst nach Monaten zurück. Mit Ausnahme einer geringen Schmerzempfindung in der Regio buccinatoria, welche noch zuweilen auftrat, war von dem frïheren Leiden nichts zurïckgebliehen. K. ging seinem früheren Gewerbe wieder nach. Er hat ein Jahr hindurch einen Stand zum Verkauf seiner Waaren inne gehabt, wo er Wind und Wetter ausgesetzt gewesen, doch seine Schmerzen blieben aus und sind nach einem Zeitraum von 7 Jahren nicht wiedergekehrt. $\mathrm{Er}$ ist demnach wohl mit Recht auf die Seite der durch die Operation Geheilten zu setzen.

Ich will nun versuchen einige der auf die Krankheit und Operationen des Morris sich beziehende Fragen zu erörtern, es dem Urtheile der besser Unterrichteten uberlassend, meinen Ansichten beizustimmen oder dieselben zu verwerfen. Ich wende mich zunächst zu den spasmodischen Erscheinungen mit nachfolgender Lähmung, welche in der letzten an demselben ausgeführten Operation stattfanden. Was war die Ursache derselben? Wir wissen, dass die 
Hauptwirkung des Chloroforms darin besteht, eine Anämie des Gehirns zu erzeugen, welcher Zustand zuweilen in Tod übergehen kann, wenn nicht Anstalten getroffen werden, diese Anämie zu beseitigen. Ich habe in meiner Praxis drei Fälle erlebt, wo der Tod positiv eingetreten war und welche sämmtlich ins Leben zurückgebracht wurden, indem sie durch Unwendung des Körpers in eine Lage gebracht wurden, worin das Blut mechanisch zum Gehirn zurücklief. (Ich werde in meiner Abhandlung über Chloroformtod darauf zurïckkommen.) Wenn wir ferner berücksichtigen, dass bei unserem Kranken die Hälfte der normalen Blutzuströmung zum Gehirn durch Unterbindung der Carotis com. abgeschnitten war, wodurch das Gehirn um $1 / 3$ seines normalen Blutgehalts beraubt worden, so können wir ỉber den Erfolg nicht weiter staunen. Ist es ja der Fortbestand der normalen Blutcirculation, wodurch die Nervencentra im Zustande functioneller Activität gehalten werden und folgeweise durch eine Unterbrechung des Maasses von Nerventhätigkeit, welche die Muskeln von den grossen Nervencentris aus regiert, müssen die Muskeln nothwendigerweise aufhören normal zu functioniren. Wir sehen dieselben in einen spasmodischen Zustand verfallen und später exlahmen. ,Schuh beobachtete eine Lähmung der rechten Körperhälfte nach einer Unterbindung der linken Carotis in Folge einer Verletzung der Maxillaris interna. Die Sprache war gehemmt, die Musculatur der rechten Körperbälfte wurde atrophisch und nach 6 Jahren paralytisch." Bei den crerebralen Hemiplegien, welche von den motorischen Centralganglien ausgehen, wird angegeben, dass sich daran nur die Muskeln der Extremitäten und des Gesichts auf der der Läsion entgegengesetzten Seite betheiligten, mit Ausnahme der Kau-, Gaumen- und Zungenmuskeln. In Morris' Fall waren auch die letzteren in einem, wenn auch bald vorübergehenden, paretischen Zustande. Patient konnte weder die Zunge herausstrecken, noch schlucken, noch in den ersten 24 Stunden ein verständliches Wort sprechen.

Ich wende mich nun zur Unterbindung der Carotis als ein Heilmittel bei Gesichtsneuralgien. Was mich nach so vielen fehlgeschlagenen Operationen veranlasste, in der Unterbindung der Carotis mein Heil zu suchen, waren wie erwähnt die Anpreisungen eines hochstehenden Chirurgen, welcher in der Unterbindung der Carotis bei Facialneuralgien die einzig wahre Panacee gefunden zu haben glaubt. Ich kann nicht leugnen, dass ich trotz dieser Recommandation mit einem gewissen Misstrauen an diese Operation ging, inden ich in allen Fällen von Facialneuralgien die Schmerzen ;abnehmen 
sah, so uft cin vermohrter Zufluss ron Blut nach den schmerzhaften Gegenden stattfand, concomitirend mit Entziindungszuständen. Irre ich mich nicht, so hat Prof. P'odrazki in Wien bei linkseitiger Facialneuralgie, ehe er die Resection des Oberkiefernerven vornahm. ebentalls die Carotis unterbunden und nicht allein ohne den gehofften Erfolg, sondern im Gegentheil nahmen die Schmerzen nach der Unterbindung' an Heftigkeit in der Wangenbeingegend zu, ja traten nun auch in der Gegend der hinteren und oberen Mahlzähne aú

Richten wir nun unsere Aufmerksamkeit auf die Resection des Facialnerven und die damit endg'îltig erzielte Heilung der Neuralgie. Für ihre Berechtigung, auch wenn keine anderen Grïnde aufgefunden werden könnten, spricht der Erfolg. Dass bei fortbestehenden Schmerzen nach gänzlicher Hinwegräumung des Oberkiefernerven und ohne dass eine Regeneration des letzteren stattgefunden, die Schmerzen in dem Facialis ihren Sitz haben mussten, dariber konnte wohl kaum ein Zweifel obwalten. (Und trotzdem liess ich mich verleiten dic Carotis zu unterbinden!) Der Nervus facialis oder die Portio dura des 7 . Nerven, wie er früher bezeichnet wurde, wurde anfünglich als ein sensibeler Nerv angesehen, bis Bell und Magendie nachwiesen, dass er ausschliesslich ein motorischer Nerv sei, welcher Ansicht auch heute Anatomen und Physiologen von grossem Rufe huldigen. Ich glaube indess, dass Gründe vorhanden sind, denjenigen Recht zu geben, welche ihn für einen gemischten Nerven betrachten. Seine Vereinigung mit dem Nerv. petrosus superf. major kann selbst mit dem Messer nachgewiesen werden. Der Facialis anastomosirt ferner mit dem unteren Maxillarnerven durch den Nerv. auriculo-temporalis, er ist verbunden mit den aus dem Ganglion oticum kommenden Nerv. petrosus superf. minor, mit welchem er am Knie des Fallopischen Kanals communicirt. Die Nervi zygomatici und buccales des 5. Nerven ramificiren mit dem Nerven gleichen Namens aus dem 7 . Nerveu kommend und die gleichnamigen Muskeln versehend. Die Aeste des Facialis, wie die zartesten Ramificationen bilden mit den Verästelungen des Oberkiefernerven Anastomosen für die Muskeln und Gefässe des Antlitzes. Können wir hiernach noch im Zweifel sein, für was wir den"Facialis zu betrachten haben? Eine vorhandene Sensibilität nach vollständiger Resection des Oberkiefernerven kann nur durch ein Vicariren des Facialnerven erklärt werden, und kann ein solches Verbältniss zwischen beiden Nerven nicht selbst bei unzerstörter Nervenbahn des Oberkiefernerren bestehen? Ein solch gegenseitiges Vertreten gewisser Nerven- 
gruppen finden wir z. B. zwischen Sympathicus und Quintus, welche sich in den Rollen als Erregungs- und Hemmungsnerven ersetzen. Die pathologischen Zustände gewisser Nerren, wie deren physiologische Wirkungen liegen noch sehr im Argen und unser Wissen in Bezug ihrer ist Stïckwerk. Sind doch die besten Beobachter noch im Streite, ob der Geschmacksinn dem Glossopharyngeus oder dem Lingualis angehört. Cla ude Bernhard räumt die beiden vorderen Drittheile der Zunge dem Lingualis, den hinteren Drittheil dem Glossopharyngeus ein. Warum sollte nicht auch zwischen Quintus und Facialis ein solches Partnership bestehen können. Der Facialis wird zwar par excellence der Nerv der Physiognomie genannt, doch kann nicht geleugnet werden, dass auch der Quintus zum Gesichtsausdruck in näherer Beziehung steht, wie dies in dem Falle von Morris in so eclatanter Weise sich zeigte, wo die Resection des Oberkiefernerven eine bei Weitem grössere Parese gewisser Muskeln zur Folge hatte als nach Durchschneidung des Facialis zu beobachten war. Die Ansicht Wagner's, des leider allzufruh verstorbenen Königsberger Chirurgen, dass ein leidender Nerv in der Bahn eines anderen Schmerzen hervorbringen könne, ist gewiss nicht so schwer zu begreifen, wenn man die vielen Anastomosen gewisser Nerven unter einander in Betracht zieht, der Vereinigung derselben in ihrem peripherischen Endigungen, von denen wir ja so viel wie nichts wissen, gar nicht zu gedenken. Wenn es noch eines weiteren Beweises bedurfte fur den innigen Zusammenhang zwischen Facialis und Quintus, so könnte man noch anfübren, dass in Facialislälmungen der galvanische Reiz auf die gelähmten Muskeln Reflexerscheinungen im Gebiete des Quintus, wie Lachkrämpfe hervorbringe. Was die Entstellung des Gesichtszlige nach Durchschneidung des Facialis anbetrifft (bekanntlich der Hauptgrund, welcher gegen die Operation angeführt wird), so ist es damit, wie wir gesehen haben, nicht so gefährlich. Claude Bernhard behauptet, dass bei Durchschneidung des Facialis an Thieren die Gesichtszäge der operirten Seite sich verzögen, beim Menschen dagegen das Umgekehrte stattfände, und erklärte dies dadurch, dass der Muskeltonus der operirten Seite verloren gegangen und dadurch die Antagonisten mit um so stärkerer Wirkung aufträten. Diese Ansicht hat sich in unserem Falle nicht bewährt. Es hatte hier mehr den Anschein, als ob wirklich eine Umkehrung der physiologischen Wirkungen zwischen den beiden Nerven Facialis und Quintus stattgefunden habe, so dass der letztere Träger der Motilität, ersterer dagegen Repräsentant der Sensibilität geworden. Es giebt noch eine andere Erklärungs- 
weise, warum dic Entstellung nach Durchschneiden des einen oder anderen Nerven nicht so in die Augen fällt. Wir sehen, dass nach Durchschneidung des Quintus die Secretion der Drüsen auf der entsprechenden seite nicht aufgehoben, sondern nur vermindert wird, weil die Excitation derselben Nerven der unverletzten Seite durch Reflexion auf die Druisen der verwundeten Seite wirkt; ebenso mag dies in gewissem Sinne der Fall sein mit dem Facialis, und hierdurch wird erklärlich, warum die Entstellung in der Praxis nicht so ausfällt, als dies der Theorie nach stattfinden sollte. Nach dem Vorausgehenden sehe ich mich genöthigt, Angesichts der Heilung von einer Gesichtsneuralgie durch die Resection des Facialnerven die Meinung auszusprechen, dass in solchen Fällen, nach resultatloser Resection des Infraorbitalnerven, sofort die Resection des Facialis vorgenommen werden sollte. Ich stütze diese Ansicht nicht auf einen Fall, sondern begrinde sie mit der Geschichte so vieler ungeheilter Fälle von Gesichtsneuralgien nach Resection des Infraorbitalnerven; auch glaube ich in Bezug der Resection des zweiten Astes des Quintus, dass es mit der Resection des Infraorbitalnerven in der Augenböhle mit Einschluss der Zahnnerven sein Bewenden haben könnte und dass eine weiter ausgedehnte Operation, wie Resection des Nerven am Foramen rotundum, wohl in den wenigsten Fällen angezeigt, oder von einem Werthe sein wird.

Zum Schluss noch ein Wort über Nervenregenerationen. Ich habe nie darin geglaubt, noch solche gesehen, so oft iẹh auch darnach gesucht habe. Das, was wir bei Wagner darüber finden, klingt geradezu fabelhaft. Es ist schon vorgekommen, dass man fibröse Trabeculae, die Elemente des Narbengewebes für Nerven gehalteu bat. Ist ein solcher Irrthum einmal möglich, so kann er sich auch wiederholen.

Die durch Resection des Oberkiefernerven in die Welt hinein posaunten Heilungen des Gesichtsschmerzes entbehren zum grossen Theil der Zuverlässigkeit, welche in einer viele Jahre fortgesetzten Beobachtung der Operirten allein zu finden ist. Viele Operateure und namentlich solche, welche die Operation zum ersten Mal unternommen, bestochen von dem augenblicklich günstigen Resultate ihres operativen Eingriffs, können die Zeit kaum erwarten, die Geschichte der Resectionen mit einem neuen glücklichen Falle zu bereichern. So kommt es denn, dass die frühzeitig als geheilt Erklärten nach einiger Zeit wieder kommen, um das alte Klaglied von Neuem zu singen; doch selten erfährt das wissenschaftliche Publicum diese 
569 XVIII. Schuppert, Zwei Fälle von rechtseitigem Gesichtsschmerz.

Niederlage einer verfrühten Mittheilung, welche damit endete ,der Patient wurde 14 Tage nach vollendeter Operation gebeilt entlassen. Wir haben seitdem nichts mehr von ihm gehört ". Of course not! Ich möchte daher gegen allzufrühe Publication solcher "gliicklichen Resectionen" warnen, indem sie die Resultate der Statistik noch unsicherer machen als sie sind. 